\title{
Amelioration of axis-aligned motion bias for active versus stationary judgments of bilaterally symmetric moving shapes' final destinations
}

\author{
Igor Dolgov, David A. Birchfield, Michael K. MCBeath, \\ HARVEy ThORNBurg, AND Christopher G. TODD \\ Arizona State University, Tempe, Arizona
}

\begin{abstract}
Recent research confirms that observers' judgments of projected final destinations of axis-trajectory misaligned moving figures are biased in the direction of primary axis deviation from trajectory, a phenomenon we named the axis-aligned motion (AAM) bias. The present study tests whether this bias occurs in a large, immersive mixed-reality environment that enables active (mobile) responses in making judgments of shapes' destinations. Like Morikawa (1999), we found that accuracy depended on axis-trajectory alignment and that there was a correspondence between final destination judgment error and the direction of axial deviation from the trajectory. Extending prior work, we found that comobile judgments were significantly more accurate than stationary ones for symmetric moving shapes, regardless of axial deviation, but only marginally so for asymmetric shapes. We conclude that our findings are ecologically consistent and that AAM is a natural regularity for which people have acquired a complementary perceptual-cognitive attunement: the AAM bias.
\end{abstract}

Symmetry provides a ubiquitous principle for organizing the surrounding environment. Mach (1897/1959) was one of the first scientists to consider the psychological impact of symmetry by demonstrating that viewers were more sensitive to asymmetries around the vertical axis than to those around the horizontal one. In the intervening century, symmetry perception has been widely investigated in humans and has been successfully modeled using artificial neural networks (see Tyler, 1996). Research with young infants has corroborated that they react to the presence of symmetry in visual stimuli (Bornstein, Ferdinandsen, \& Gross, 1981) and exhibit a preference for vertically symmetric figures (Bornstein \& Krinsky, 1985). Furthermore, Smith (2005) demonstrated the salience of symmetry in motion and category perception for infants, supporting the assertion that, beginning at an early age, it is used as a fundamental cue for perception and action.

Symmetry axes have been shown to play an important role in guiding visual search (Niimi, Yokosawa, \& Watanabe, 2006; Wolfe \& Friedman-Hill, 1992), establishing a visual reference frame (Palmer \& Hemenway, 1978; Sekuler \& Swimmer, 2000), categorizing shapes and determining their identity (McBeath, Schiano, \& Tversky, 1997; Smith, 2005), form perception and shape constancy (Freyd \& Tversky, 1984; Pizlo \& Stevenson, 1999), segmenting complex figures into component parts (De Winter \& Wagemans, 2006), perceiving centers within a shape (Davi, Doyle, \& Proffitt, 1992), object-orientation processing (Boutsen \& Marendaz, 2001; Wenderoth, 1997), and general perceptual organization (Arnheim, 1974;
Piaget, 1969), among others. ${ }^{1}$ Research also confirms that symmetry axes are highly salient cues in rotational (Farrel \& Shepard, 1981; Pani, William, \& Shippey, 1995) and translational (Dolgov, McBeath, \& Sugar, 2005; McBeath, Morikawa, \& Kaiser, 1992; Morikawa, 1999) motion perception. In the present experiment, we explore how bilateral symmetry impacts the perception of linearly moving symmetric and asymmetric geometric shapes projected onto the floor of an immersive mixed-reality environment.

Work by Morikawa (1999) and Dolgov et al. (2005) confirmed that observers exhibit behavior in a manner consistent with the assumption that bilaterally symmetric moving objects maintain bilateral axis alignment with their trajectory, a perceptual regularity that we named the axis-aligned motion (AAM) bias. If axis-trajectory alignment is maintained, observers' trajectory judgments are highly accurate; otherwise, judgments of final destination and trajectory are biased in the direction of axial deviation (see Figure 1).

The AAM bias is a complementary attunement of the perceptual-cognitive system to the natural regularity of axis-trajectory alignment maintained during locomotion by nearly all animate entities (Hargittai \& Hargittai, 1994; McBeath et al., 1992; Morikawa, 1999). In an ecological context, having this bias is generally advantageous. ${ }^{2}$ However, in the modern world, the bias can frequently have a negative impact, because artifacts (e.g., some vehicles, tools, and projectiles) are not always constrained by the AAM regularity.

In five experiments, Morikawa (1999) demonstrated the existence of an AAM bias for judging the motion direc- 


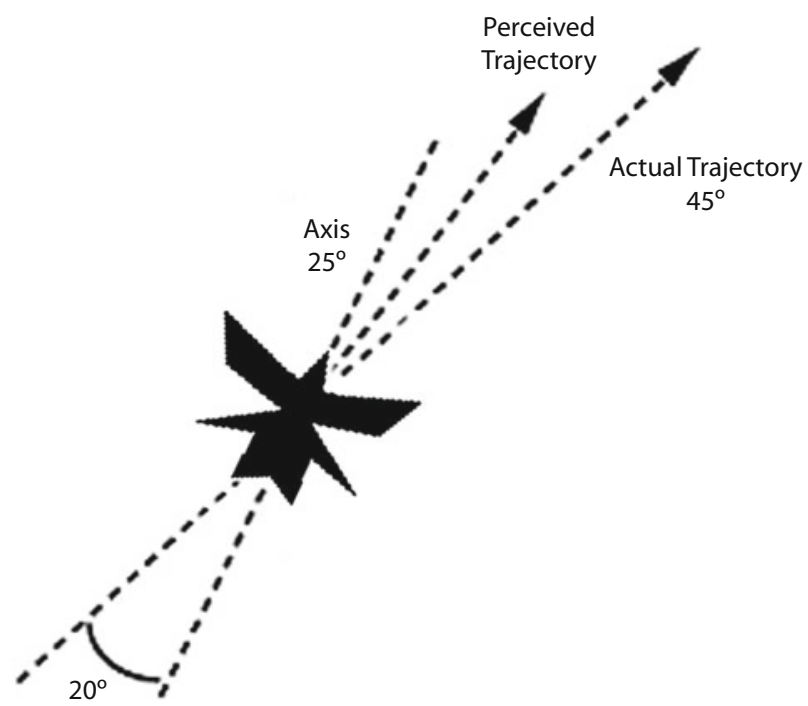

Figure 1. The target is moving on a trajectory that is $45^{\circ}$ from vertical, but it is oriented with its axis $25^{\circ}$ from vertical. This configuration results in a perception that the target shape is moving on a trajectory that is somewhere between the actual trajectory and the axial orientation. Diagram adapted from Morikawa (1999), Figure 1; also see his Figures 4 and 5 for plots of perceived versus actual axial deviation. Copyright 1999, Psychonomic Society, Inc. Adapted with permission.

tion of linearly moving geometric shapes displayed on a 2-D monitor. He found that, when an object's primary axis was established by bilateral symmetry (Experiments 1-3) or elongation (Experiment 5), axial deviation away from trajectory resulted in systematically biased judgments of trajectory (Experiments 1,3, and 5) and of final destination (Experiment 2). Consistent with Johnstone and Wenderoth (1989), the opposite was not true, with motion having no effect on perceived orientation (Experiment 3). Furthermore, due to the absence of a primary axis, no systematic perceptual biases were observed for nonelongated asymmetric shapes (Experiments 1, 2, and 5). When motion of symmetric shapes was axis aligned (ecologically consistent), participants made judgments significantly faster (Experiment 4) and more accurately than they did for motion of asymmetric shapes, whose destinations in all experiments were judged significantly more accurately than were those of axis-trajectory misaligned symmetric shapes.

The present study extended the 2-D experimental procedure used by Morikawa (1999) in his second experiment to a novel perceptual setting: the Situated Multimedia Arts Learning Laboratory (SMALLab), an immersive mixedreality environment. We chose the final destination estimation task from Morikawa's second experiment because of his commendation of its ecological validity. In general, our goal was to study the AAM bias in a quasirealistic setting, while retaining the measurement precision offered by technology. The large, immersive nature of the SMALLab environment also allowed us to examine the impact of different judgment methods on participants' accuracyspecifically, by comparing stationary (pointing-based) and active (locomotion-based) methods of judging the final destinations of floor-projected moving geometric shapes.

Our hypotheses were twofold. Following Morikawa (1999) and Dolgov et al. (2005), we expected participants to experience an AAM bias in judgments of symmetric shapes. Consequently, we hypothesized that judgment error would be predicted by symmetric shapes' axial deviation from trajectory (AAM bias hypothesis). Our secondary hypothesis was motivated by prior research that showed that perceptual biases and illusions frequently disappear when experimental task parameters are changed so that participants perform an active rather than a passive judgment (Carey, 2001; Milner \& Goodale, 1992, 1993, 1995; Post \& Welch, 1996). We hypothesized that increased action system engagement would improve performance (action hypothesis).

\section{METHOD}

\section{Participants}

Eight (5 male, 3 female) undergraduates from Arizona State University's psychology subject pool participated and received course credit as compensation. All had normal or corrected-to-normal vision.

\section{Apparatus}

The SMALLab uses a network of computers that track lighted objects and provide audiovisual stimuli and feedback for an immersive multimedia classroom environment (Birchfield, Ciufo, \& Minyard, 2006). All of the sensing and feedback hardware (e.g., cameras, IR system, projector, speakers, power and network cables) is mounted on a $5 \times 5 \times 4$ m truss support structure (see Figure 2 ).

The visual tracking computer is connected to six digital video cameras (three video, three infrared) and uses a proprietary colorand-infrared tracking algorithm (Jin \& Qian, 2007; Jin, Qian, \& Rajko, 2006) to locate lighted objects with precision on the order of $2 \mathrm{~mm}$. Visual stimuli are generated on the interaction computer and are projected (NEC VT660, 1,700 lumens) onto a lightly padded white floor by being reflected in a mirror mounted along the top of the truss structure. A proprietary calibration algorithm synchronizes the visual tracking and interaction computers (Jin, Qian, \& Birchfield, 2008) to account for any visual distortions. To indicate final destination, participants used a lighted green ball that was $5 \mathrm{~cm}$ in diameter and was attached to a small rectangular base that allowed it to rest on the floor without rolling.

\section{Procedure}

To enhance tracking and ensure the visibility of the projected shapes and background, direct overhead lighting was slightly dimmed. The experimental environment was projected onto the floor. It consisted of a central ring (3.5-m diameter) that signified the main experimental space in which the stimuli would appear and move, and smaller rings $(40-\mathrm{cm}$ diameter) in each of the corners that signified possible observer starting locations (see Figure 3). Participants observed moving geometric shapes that traveled at $1 \mathrm{~m} /$ $\mathrm{sec}$ and were approximately $25 \mathrm{~cm}$ long. Two symmetric and two asymmetric shapes (see Figure 4) were selected from a collection used by Morikawa (1999) that was originally generated by Farrel and Shepard (1981) and had been further developed by McBeath et al. (1997).

Each observer starting position (which remained the same within a block) corresponded to a unique quasirandom ordering of the trials, in which each shape was presented six times in a 24-trial block. At the beginning of each trial, a shape appeared at one of the starting locations on the central ring (Points $\mathrm{W}-\mathrm{Z}$ in Figure 4) and moved across the experimental space, disappearing approximately half- 


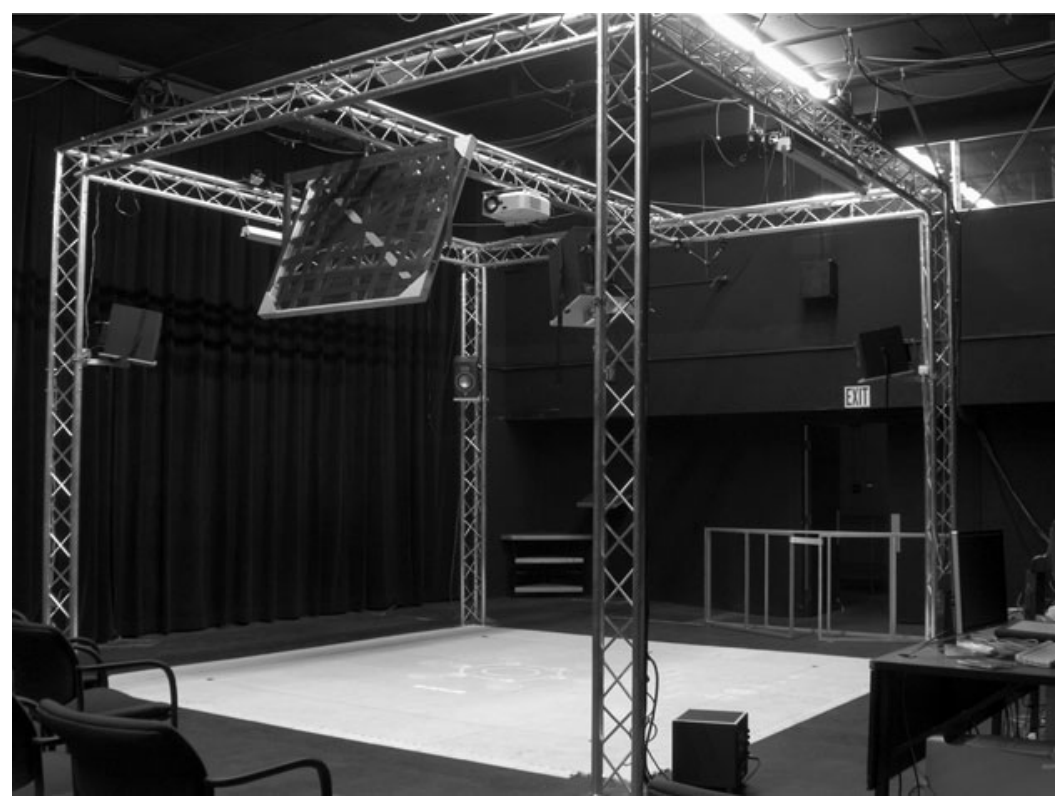

Figure 2. Photograph of the SMALLab environment, which is housed in the Digital Arts Ranch, a converted black box theater on the Arizona State University campus. Typically, the lighting directly above the SMALLab is dimmed so that the projected image is vivid on the white floor mat, and the ambient theater lighting remains unchanged.

way across the ring (Points A-E in Figure 3). Similar to Morikawa's (1999) design, symmetric shapes were presented with axes either aligned to trajectory or deviating from trajectory by $\pm 30^{\circ}$; asymmetric shapes were assigned an arbitrary axis and underwent the same transformations. Each shape was presented twice at each axial deviation. In order to minimize memorization of specific trajectories, shape trajectory and shape origin were counterbalanced. Axial deviation was also counterbalanced across trajectories, in such a way that each trajectory was traversed an equal number of counterclockwise, clockwise, and nondeviated shapes. In order to best approximate an ecologically valid pursuit scenario, we limited shape starting location to the two possible starting points adjacent to the participant's starting location.

After the shapes disappeared, the participants judged their projected destinations as if they had continued moving and terminated at the boundary of the central ring. Participants indicated their perceived final destinations by placing the lighted ball at the appropriate spot on the floor. In the active (mobile) conditions, the participants completed this task by themselves; in the stationary condition, the participants used a laser pointer to indicate the perceived destination, at which an experimenter's assistant placed the ball.

Participants completed three 24-trial blocks, one for each experimental condition: (1) stationary, in which participants estimated shapes' destinations using a laser pointer; (2) postmobile, in which participants walked to destinations after the shapes disappeared; and (3) comobile, in which participants walked concurrently with the moving shapes. To avoid shadowing problems with the video projection, participants always performed the comobile condition at Observer Starting Location 3 or 4, at which shadowing was not a problem. Six possible block order sequences were generated, one of which was assigned randomly to a participant at the beginning of the experimental session.

\section{Design}

The dependent variable in all analyses was judgment error, measured in degrees of angular deviation of the judgment from the actual final destination of the moving shape along the central ring (com- puted in MATLAB, using simple trigonometry). To reduce systematic biases due to possible projection and/or measurement error, data were collapsed for each participant by averaging trials in each cell of the fully crossed design.

Error directionality was assessed via a two-factor ANOVA that examined only judgments of symmetric shapes and used signed values of judgment error for the dependent variable. We employed two fully crossed within-subjects factors: judgment method (3: stationary, postmobile, comobile) and axial deviation (3: counterclockwise, $-30^{\circ}$; none, $0^{\circ}$ : clockwise, $+30^{\circ}$ ). Due to the averaging of positive and negative errors in this analysis, we anticipated judgment method to be insignificant.

The error magnitude analysis quantified performance as a function of two within-subjects factors: judgment method (3: stationary, postmobile, comobile) and shape symmetry type (SST; 3 : symmetric, axis-trajectory aligned; asymmetric; and symmetric, axistrajectory misaligned) and used absolute values of judgment error as the dependent measure. We employed one fully crossed ANOVA with several subsequent $3 \times 1$ ANOVAs and pairwise comparisons (significance was Šidák adjusted throughout) to evaluate the interaction and simple main effects.

\section{RESULTS}

Both analyses revealed strong support for the AAM bias hypothesis. The error directionality ANOVA revealed a highly significant effect of axial deviation $[F(1.2,9.5)=$ 21.6, $p<.005] .{ }^{3}$ Participants' errors corresponded to the axial tilt of the shapes across all judgment methods, with observers exhibiting judgment error means of $-7.4^{\circ}$, $-0.4^{\circ}$, and $7.6^{\circ}$ for counterclockwise-deviated, nondeviated (axis-aligned), and clockwise-deviated shapes, respectively. Pairwise comparisons revealed a significant difference in judgment error between clockwise-deviated and nondeviated shapes $(p<.001)$ and also between the 


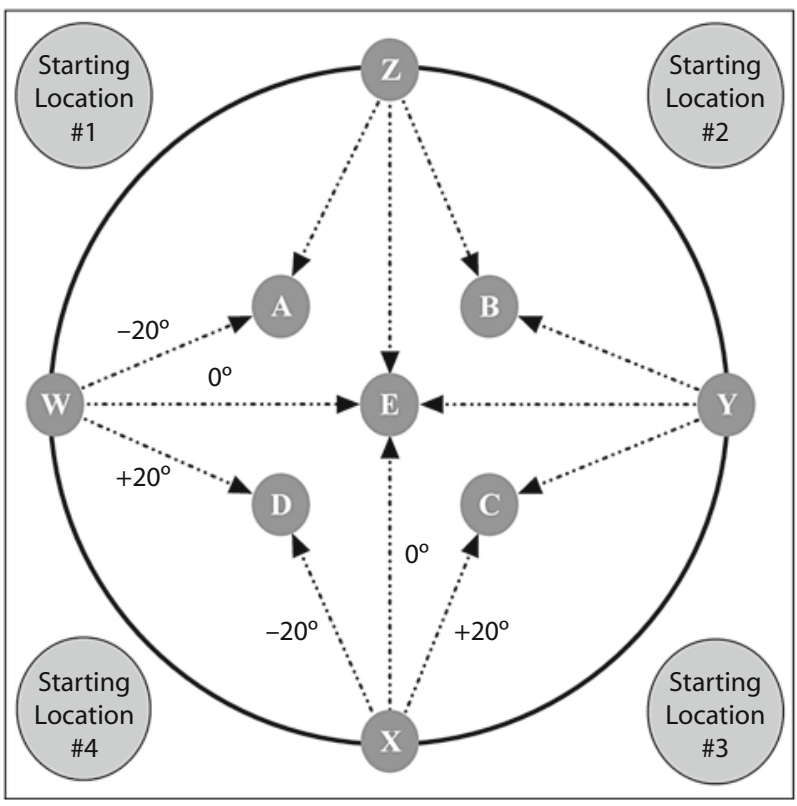

Figure 3. Diagram of the experimental space showing: Central ring, four observer starting locations (1-4), four shape starting locations $(W-Z)$, and five disappearing points $(A-E)$. Only the central ring and four smaller starting location rings remained visible throughout the experiment. The paths and locations $(A-E$ and $\mathrm{W}-\mathrm{Z}$ ) were never visible nor identified in any way. Participants were asked to face the middle of the central ring at the beginning of each trial.

clockwise- and counterclockwise-deviated shapes $(p<$ $.005)$. As we predicted for this analysis, there was no effect of judgment method $[F(2,14)=1.5$, n.s. $]$ due to the averaging of positive and negative judgment error values; means were $0.9^{\circ}, 0.2^{\circ}$, and $-1.3^{\circ}$ for the stationary, postmobile, and comobile conditions, respectively. The interaction also was not significant $[F(4,32)=1.6$, n.s.].

In further support of the AAM bias hypothesis, the error magnitude analysis revealed a highly significant main effect of SST $[F(1.1,8.0)=24.7, p<.001] .{ }^{4}$ However, there was also a significant interaction between SST and judgment method $[F(4,28)=2.8, p<.05]$ (see Figure 5). We explored the interaction by examining the simple main effects of SST by conducting $3 \times 1$ ANOVAs at each level of judgment method. We found a significant effect of SST in each judgment method [stationary, $F(2,14)=20.6, p<$ .001 ; postmobile, $F(1.1,7.7)=12.2, p<.01 ; 5$ comobile, $F(2,14)=17.3, p<.01]$, with subsequent pairwise comparisons elucidating the differences in performance for each SST.

Judgments of symmetric aligned shapes were significantly more accurate than were those of symmetric misaligned shapes $(p s<.05)$ across all judgment methods, thus supporting the AAM bias hypothesis. In the stationary and postmobile conditions, the results mirrored those of Morikawa (1999): Symmetric misaligned shapes and asymmetric shapes were significantly different $(p$ s $<$ .01 ), whereas asymmetric shapes and symmetric aligned shapes were not (although the difference was margin- ally significant in the postmobile condition). This pattern was reversed in the comobile condition, with symmetric misaligned shapes and asymmetric shapes being approximately equal, whereas asymmetric shapes and symmetric aligned shapes were significantly different $(p<.05)$. Therefore, performance in the stationary and postmobile methods most resembled that in Morikawa. However, Morikawa's observed error magnitude was less than half of what we observed for asymmetric shapes and symmetric aligned shapes in these two conditions. We believe that this discrepancy may reflect an increased difficulty of performing the final destination estimation task in a large immersive mixed-reality environment, as compared with performing the task using a standard monitor. Additionally, Morikawa's participants completed roughly 100 more trials using only one judgment method and therefore may have improved somewhat with practice. Finally, the plane of motion was changed from vertical to horizontal.

In the comobile condition, which is consistent with ecological pursuit behavior, performance was greatly aided by the presence of a bilateral symmetry axis that accurately indicated trajectory. Although this finding was not predicted a priori, it is not surprising when one considers humans' evolutionary need for the pursuit of prey and avoidance of predators, both of which are typically symmetric creatures whose bilateral axis veridically indicates their motion direction. Furthermore, interactions with genuinely asymmetric moving objects or symmetric misaligned moving objects have only recently become more frequent due to the presence of technology. Therefore, it is also not surprising that the visual system is less adept in making trajectory judgments of nonecological shapes (axis misaligned/asymmetric) in an ecologically valid judgment task like that in the comobile condition.

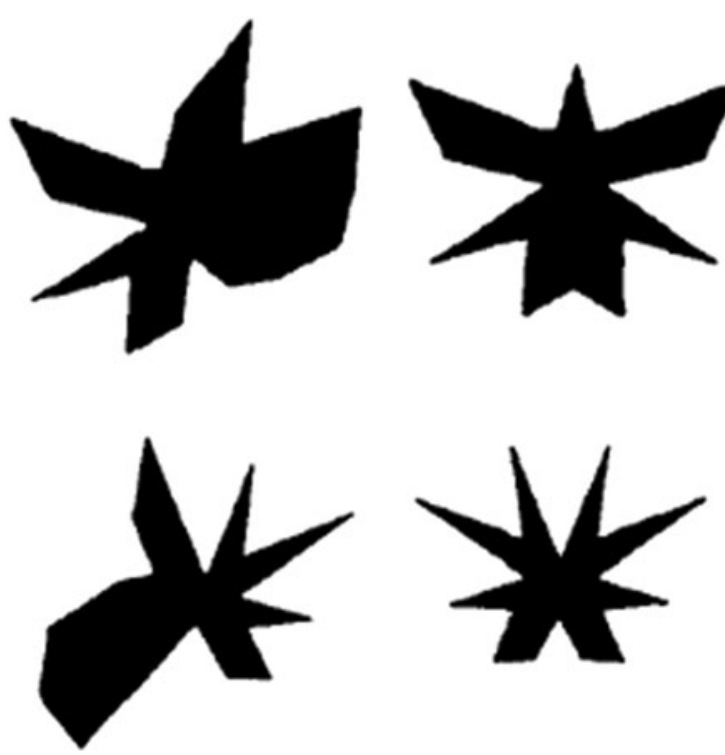

Figure 4. The abstract geometric shapes were chosen out of a subset generated by McBeath, Schiano, and Tversky (1997). The top two shapes were also used by Morikawa (1999) in his experiments. 
Performance in Final Destination Judgment Task

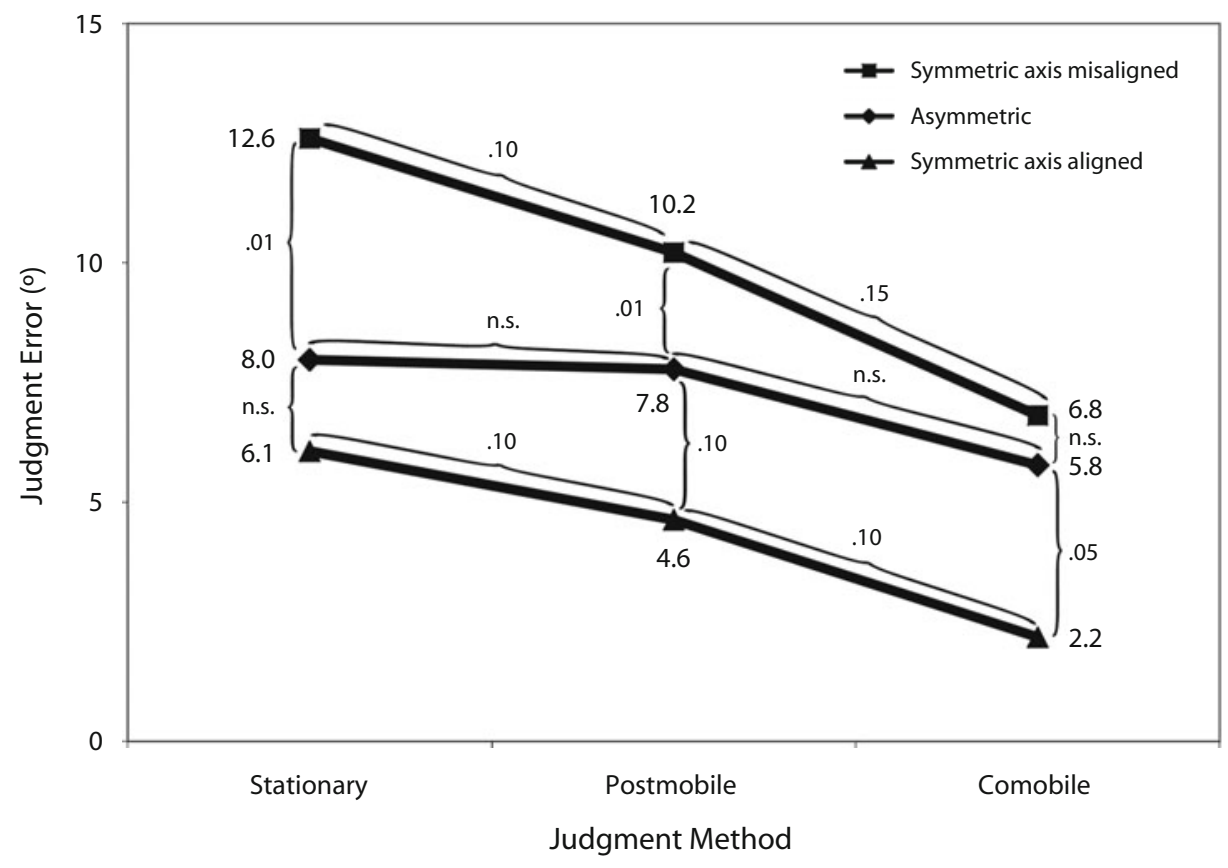

Figure 5. We found a significant interaction between judgment method and shape symmetry type. Simple main effects of shape symmetry type revealed that judgments of symmetric misaligned shapes were significantly less accurate than symmetric aligned shapes across all levels $(p \mathrm{~s}<.05$ or .01 ), and are therefore not illustrated, to reduce clutter. Simple main effects of judgment method revealed that stationary judgments were significantly less accurate than comobile judgments for symmetric shapes $(p \mathrm{~s}<.01)$ and marginally less accurate for asymmetric shapes $(p<.1)$; again, to reduce clutter, these comparisons were not shown. Šidák-adjusted significance values are provided for all other pairwise comparisons.

Although we observed a highly significant effect of judgment method $[F(2,14)=14.7, p<.001]$, in order to correctly evaluate the action hypothesis, we explored the simple main effects. We conducted three $3 \times 1$ ANOVAs, one at each level of SST. We found a significant effect of judgment method at all levels [symmetric misaligned, $F(2,14)=10.8, p<.005$; asymmetric, $F(2,14)=4.2$, $p<.05$; symmetric aligned, $F(2,14)=14.1, p<.001]$. For symmetric shapes, regardless of axis-trajectory alignment, pairwise comparisons revealed that accuracy using the comobile judgment method was significantly improved over that using the stationary judgment method $(p<.01)$, whereas the difference just approached significance for asymmetric shapes $(p<.1)$.

The improved accuracy observed in the more active judgment methods likely is due to an overall increase in engagement of the perception-action system, with the comobile method also benefiting from proximity to target. We can estimate the overall contribution of proximity by looking at the difference in performance between the postmobile and comobile conditions in judgments of asymmetric shapes, judgments that are not affected by AAM bias. This difference amounts to $2^{\circ}$ and is not significant in a pairwise comparison. On the other hand, the differences for symmetric aligned and misaligned shapes were $2.4^{\circ}$ and $3.4^{\circ}$, respectively, and both approached statistical significance $(p<.06, p<.15)$, suggesting an overall contribution of action beyond that of proximity using the comobile judgment method for symmetric shapes. Furthermore, a similar pattern is also present when we compare performance between the stationary and postmobile judgment methods, where there are no potential contributions of proximity. For asymmetric shapes, the difference was less than half of a degree and was not significant. For symmetric aligned and misaligned shapes, the differences were $1.5^{\circ}$ and $2.4^{\circ}$, respectively, and both approached significance ( $p \mathrm{~s}<.1$ ). Altogether, the action hypothesis was strongly supported only for the juxtaposition of stationary and comobile judgment methods, although there is evidence that overall increased engagement does ameliorate the bias for symmetric shapes.

Although we currently cannot draw general conclusions about the action hypothesis, our results exhibit a pattern consistent with those of prior research, which shows that active perceptual judgment tasks are less susceptible to illusions than are tasks that are passive and are typically thought of as purely perceptual (e.g., Carey, 2001; Post \& Welch, 1996). Such findings are not surprising in light of the fact that humans evolved as active, mobile creatures and employ a variety of navigation (Gibson, 1966, 1979) and interception (Sugar, McBeath, \& Wang, 2006) strategies. We plan to empirically assess the use of pursuit 
strategies in making final destination judgments by recording trajectories of both participants and targets using a marker-based Vicon motion-capture system in an upcoming large-scale, real-world experiment.

\section{DISCUSSION}

This study confirmed that people experience an AAM bias when judging final destinations of floor-projected moving geometric shapes in a large, immersive mixedreality environment. Although the term bias typically implies a phenomenon that always entails some perceptual disadvantage, the AAM bias is actually a mixed blessing whose true impact depends on axis-trajectory alignment. When the axis of a moving symmetric shape is misaligned with respect to its trajectory, observers' judgments of final destination are biased by axial deviation. However, in the ecologically consistent case, when the axis is aligned with its trajectory, an AAM bias works to the observers' advantage. Additionally, we have shown that engaging the action system may ameliorate the impact of AAM bias, a finding consistent with prior object motion perception literature that compares stationary and active judgment methods (e.g., Oberle, Hollums, McBeath, \& Terry, 2006).

Shepard $(1987,1994)$ pointed out that psychological mechanisms are perceptual-cognitive reflections of highly reliable natural regularities in the physical world. If life forms have tended to exhibit AAM throughout the bulk of evolutionary history (which is highly likely, given the skeletal structure of once animate fossils), having an AAM bias would have been ecologically advantageous for any mobile animal. A simple mechanism that improves an organism's interceptive/avoidance abilities increases its Darwinian fitness and would therefore favor its being naturally selected. Regardless of its origins, the pragmatic realization of AAM bias is an often advantageous predictive mechanism for judging the trajectory of moving objects with a salient primary axis. We conclude that although the AAM bias is a two-edged sword, it is generally a beneficial perceptual mechanism for quickly and accurately judging the direction of the ecologically consistent moving entities that populate our environment.

\section{AUTHOR NOTE}

This research was supported by NSF Grant CISE-0403428. The authors thank the NSF, Arizona State University, the Department of Psychology, and the Arts, Media, and Engineering Program for providing and encouraging a fertile academic environment and enabling our research. We also thank the reviewers, editors, and our significant others who have worked diligently to improve this work. Address correspondence to I. Dolgov, Department of Psychology, Arizona State University, Box 871104, Tempe, AZ 85287-1104 (e-mail: id@asu.edu).

\section{REFERENCES}

Arnheim, R. (1974). Art and visual perception: A psychology of the creative eye. Berkeley: University of California Press.

Birchfield, D., Ciufo, T., \& Minyard, G. (2006). SMALLab: A mediated platform for education. In Proceedings of International Conference on Computer Graphics and Interactive Techniques: ACM SIGGRAPH 2006 Educators Program, Boston. Retrieved July 26, 2008, from portal.acm.org/citation.cfm?id=1179295.1179329
Bornstein, M. H., Ferdinandsen, K., \& Gross, C. G. (1981). Perception of symmetry in infancy. Developmental Psychology, 17, 82-86.

BornsteIn, M. H., \& KRINSKY, S. J. (1985). Perception of symmetry in infancy: The salience of vertical symmetry and the perception of pattern wholes. Journal of Experimental Child Psychology, 39, 1-19.

Boutsen, L., \& Marendaz, C. (2001). Detection of shape orientation depends on salient axes of symmetry and elongation: Evidence from visual search. Perception \& Psychophysics, 63, 404-422.

CAREY, D. P. (2001). Do action systems resist visual illusions? Trends in Cognitive Sciences, 5, 109-113.

Davi, M., Doyle, M. A., \& Proffitt, D. (1992). The role of symmetry in determining perceived centers within shapes. Perception \& Psychophysics, 52, 151-160.

De Winter, J., \& Wagemans, J. (2006). Segmentation of object outlines into parts: A large-scale integrative study. Cognition, 99, 275-325.

Dolgov, I., McBeath, M. K., \& Sugar, T. G. (2005). The influence of symmetry on perception of thrown, oblong, symmetrical projectiles in 3D. In H. Heft \& K. L. Marsh (Eds.), Studies in perception \& action VIII (pp. 132-136). London: Routledge.

Farrel, J., \& ShePard, R. (1981). Shape, orientation, and apparent rotational motion. Journal of Experimental Psychology: Human Perception \& Performance, 7, 477-486.

Freyd, J., \& TVERSKY, B. (1984). Force of symmetry in form perception. American Journal of Psychology, 97, 109-126.

Gibson, J. J. (1966). The senses considered as perceptual systems. Boston: Houghton Mifflin.

GiBson, J. J. (1979). The ecological approach to visual perception. Hillsdale, NJ: Erlbaum.

Hargittai, I., \& Hargittai, M. (1994). Symmetry: A unifying concept. Bolinas, CA: Shelter Publications.

JAYNE, B. C. (1988). Muscular mechanisms of snake locomotion: An electromyographic study of the sidewinding and concertina modes of Crotalus cerastes, Nerodia fasciata and Elaphe obsolete. Journal of Experimental Biology, 140, 1-33.

JiN, H., \& QIAN, G. (2007). Robust multi-camera 3D people tracking with occlusion handling. Proceedings of IEEE International Conference on Acoustics, Speech, \& Signal Processing, 1, 909-912.

Jin, H., Qian, G., \& Birchfield, D. A. (2008, January). Real-time multi-view object tracking in mediated environments. In Proceedings of ACM International Multimedia Modeling Conference, Kyoto.

JiN, H., QIAN, G., \& RaJKo, S. (2006, November). Real-time multi-view $3 \mathrm{D}$ object tracking in cluttered scenes. In Proceedings of International Symposium on Visual Computing, Lake Tahoe, NV.

Johnstone, S., \& Wenderoth, P. (1989). Pattern orientation, not motion, determines the two-dimensional tilt illusion. Perception, 18, 729-737.

MACH, E. (1959). The analysis of sensations, and the relation of the physical to the psychical (C. M. Williams, Ed.). New York: Dover. (Original work published 1897)

McBeath, M. K., Morikawa, K., \& Kaiser, M. K. (1992). Perceptual bias for forward-facing motion. Psychological Science, 3, 362-367.

McBeath, M. K., Schiano, D. J., \& Tversky, B. (1997). Threedimensional bilateral symmetry bias in judgments of figural identity and orientation. Psychological Science, 8, 217-223.

Milner, A. D., \& Goodale, M. A. (1992). Separate visual pathways for perception and action. Trends in Neurosciences, 15, 20-25.

Milner, A. D., \& Goodale, M. A. (1993). Visual pathways to perception and action. In T. P. Hicks, S. Molotchnikoff, \& T. Ono (Eds.), Progress in brain research (Vol. 95, pp. 317-337). Amsterdam: Elsevier.

Milner, A. D., \& Goodale, M. A. (1995). The visual brain in action. Oxford: Oxford University Press.

Morikawa, K. (1999). Symmetry and elongation of objects influence perceived direction of translational motion. Perception \& Psychophysics, 61, 134-143.

Nitmi, R., Yokosawa, K., \& Watanabe, K. (2006). Attentional set for axis of symmetry in symmetry-defined visual search. Perception \& Psychophysics, 68, 1153-1162.

Oberle, C. D., Hollums, N. K., McBeath, M. K., \& Terry, D. P. (2006). Motion by nearby players biases perception but not action in judgments of baseball destination. Perceptual \& Motor Skills, 103, 585-606.

Palmer, S. E., \& Hemenway, K. (1978). Orientation and symmetry: Ef- 
fects of multiple, rotational, and near symmetries. Journal of Experimental Psychology: Human Perception \& Performance, 4, 691-702.

Pani, J. R., William, C. T., \& ShipPEy, G. T. (1995). Determinants of the perception of rotational motion: Orientation of the motion to the object and to the environment. Journal of Experimental Psychology: Human Perception \& Performance, 21, 1441-1456.

THE PERCEPTION OF SYMMETRY: EMPIRICAL ASPECTS [Special issue] (1995). Spatial Vision, 9(1).

THE PERCEPTION OF SYMMETRY: THEORETICAL ASPECTS [Special issue] (1994). Spatial Vision, 8(4).

Piaget, J. (1969). The mechanisms of perception. New York: Basic Books.

Pizlo, Z., \& Stevenson, A. K. (1999). Shape constancy from novel views. Perception \& Psychophysics, 61, 1299-1307.

Post, R. B., \& Welch, R. B. (1996). Is there dissociation of perceptual and motor responses to figural illusions? Perception, 25, 569-581.

SekUler, A. B., \& Swimmer, M. B. (2000). Interactions between symmetry and elongation in determining reference frames for object perception. Canadian Journal of Experimental Psychology, 54, 42-55.

SHEPARD, R. N. (1987). Toward a universal law of generalization for psychological science. Science, 237, 1317-1323.

SHEPARD, R. N. (1994). Perceptual-cognitive universals as reflections of the world. Psychonomic Bulletin \& Review, 1, 2-28.

Sмiтн, L. B. (2005). Action alters shape categories. Cognitive Science, 29, 665-679.

Sugar, T. G., McBeath, M. K., \& Wang, Z. (2006). A unified fielder theory for interception of moving objects either above or below the horizon. Psychonomic Bulletin \& Review, 13, 908-917.

TYLER, C. W. (ED.) (1996). Human symmetry perception and its computational analysis. Utrecht: VSP.
Wenderoth, P. (1997). The role of implicit axes of bilateral symmetry in orientation processing. Australian Journal of Psychology, 49, 176-181.

Wolfe, J. M., \& Friedman-Hill, S. R. (1992). On the role of symmetry in visual search. Psychological Science, 3, 194-198.

WootTon, R. J. (1999). Invertebrate paraxial locomotory appendages: Design, deformation and control. Journal of Experimental Biology, 202, 3333-3345.

\section{NOTES}

1. Many of the cited works provide extensive literature reviews on symmetry perception and related topics. For an excellent and insightful summary, see Freyd and Tversky (1984), and, for a broader perspective, see Tyler (1996) and the special issues of Spatial Vision (Perception of Symmetry, 1994, 1995).

2. Exceptions include scenarios in which one needs to ascertain the motion direction of unusually moving animals such as crabs (Wootton, 1999) and sidewinding snakes (Jayne, 1988).

3 . Mauchly's test indicated that the assumption of sphericity had been violated $\left[\chi^{2}(2)=8.0, p<.05\right] ; d f$ s were corrected using GreenhouseGeisser estimates of sphericity $(\varepsilon=.59)$.

4. Mauchly's $\left[\chi^{2}(2)=8.5, p<.05\right] ; d f$ s were corrected using Greenhouse-Geisser estimates of sphericity $(\varepsilon=.57)$.

5. Mauchly's $\left[\chi^{2}(2)=10.5, p<.01\right]$; $d f$ s were corrected using Greenhouse-Geisser estimates of sphericity $(\varepsilon=.55)$.

(Manuscript received June 11, 2008; revision accepted for publication September 26, 2008.) 